\title{
Optimal Vibration Stimulation to the Neck Extensor Muscles Using Hydraulic Vibrators to Shorten Saccadic Reaction Time
}

\author{
Katsuo Fujiwara ${ }^{1)}$, Kenji Kunita ${ }^{2)}$, Naoe Furune ${ }^{1)}$, Kaoru Maeda ${ }^{1)}$, \\ Hitoshi Asai $^{1)}$ and Hidehito Tomita ${ }^{1)}$ \\ 1) Department of Human Movement and Health, Graduate School of Medical Science, Kanazawa University \\ 2) Research Center for Urban Health and Sports, Osaka City University
}

\begin{abstract}
Optimal vibration stimulation to the neck extensor muscles using hydraulic vibrators to shorten the saccadic reaction time was examined. Subjects were 14 healthy young adults. Visual targets (LEDs) were located $10^{\circ}$ left and right of a central point. The targets were alternately lit for random durations of 2-4 seconds in a resting neck condition and various vibration conditions, and saccadic reaction times were measured. Vibration amplitude was $0.5 \mathrm{~mm}$ in every condition. The upper trapezius muscles were vibrated at $40,60,80$, and $100 \mathrm{~Hz}$ in a sub-maximum stretch condition in which the muscles were stretched at $70 \%$ of maximum stretch. In addition, the muscles were vibrated at $60 \mathrm{~Hz}$ with the muscles maximally stretched, with $70 \%$ vertical pressure without stretching, and with vibration applied to the skin in the same area as the muscle vibration. At 60,80 , and $100 \mathrm{~Hz}$ at $70 \%$ maximum stretch, saccadic reaction time shortened significantly compared with the resting neck condition. However, no significant difference in the reaction time was observed among the frequencies. The saccadic reaction times in the maximum stretch condition, muscle pressure condition, and skin contact condition did not differ significantly from that in the resting neck condition. Vibration stimulation to the trapezius with $60-100 \mathrm{~Hz}$ frequencies at $0.5 \mathrm{~mm}$ amplitude in the sub-maximum stretch condition was effective for shortening saccadic reaction time. The main mechanism appears to be Ia information originating from the muscle spindle. J Physiol Anthropol 25(5): 345-351, 2006 http:// www.jstage.jst.go.jp/browse/jpa2

[DOI: 10.2114/jpa2.25.345]
\end{abstract}

Keywords: saccadic reaction time, trapezius, muscle vibration, brain activation, afferent information

\section{Introduction}

Human beings are capable of maintaining a variety of postures, from which a suitable posture is selected for intended exercise. Howorth (1946) observed that a basic dynamic posture in which the foot, hip, knee and neck joints and the trunk were slightly flexed is consistently shown throughout a variety of exercises. He suggested from a biomechanical point of view that muscle tension, balance, and co-ordination of motion were optimized by maintaining this posture. Subsequently, it was demonstrated that maintaining the neck flexion position that constitutes part of the basic dynamic posture leads to the development of the tonic neck reflex and neck-eye reflex (Fukuda, 1957), shortening of reaction time (Kinugasa et al., 1985; Fujiwara, 1994), and improvement in the accuracy of pursuing a moving visual target (Hinoki et al., 1975; Fujiwara, 1994). It was reported that sensory information from muscle spindles in the neck extensors would appear to contribute greatly to these phenomena. Therefore, knowledge of the stimulus effect on the muscle spindles is necessary to understand basic postural behavior and its fundamental control mechanisms. This study was particularly interested in the relationship between activation of the central nervous system and increase in information from muscle spindles.

It was previously reported that saccadic reaction times were shortened in association with activation of the neck extensor muscles through neck flexion or moderate isometric contraction of the shoulder girdle elevators (Kunita and Fujiwara, 1996; Fujiwara et al., 2000). Possible physiological factors accounting for this shortening may be ascending brain activation mainly from the brain stem, which contains the reticular formation, or descending activation from the cerebral cortex in accordance with neck muscle action (Taniguchi et al., 1980; Grantyn and Berthoz, 1987; Vallar et al., 1995; Berthoz, 1996; Shimura and Kasai, 2002).

We previously investigated saccadic reaction time during vibration stimulation to the neck extensor muscles and found conclusive evidence of ascending brain activation (Fujiwara et al., 2001). In that study, the following research was referred to. 
The tonic vibration reflex was generated by the discharge of the muscle spindle primary endings (Steyvers et al., 2003). In the spinal frog, the response of the primary endings was tuned to the vibration frequency of $20-110 \mathrm{~Hz}$ (Giszter and Kargo, 2002). In humans, the discharge of the primary endings increased in proportion to a vibration frequency of $70-100 \mathrm{~Hz}$ (Roll et al., 1990). Eklund and Hagbarth (1966) reported that the tonic vibration reflex during vibration stimulation ceased when muscle length was maximum. It is confirmed that the amount of Ia discharge that occurs in accordance with muscle vibration increases remarkably under sub-maximum stretch conditions (Hagbarth and Vallbo, 1968; Burke et al., 1976a, b). Therefore, in our previous study (Fujiwara et al., 2001), the pressure of the vibrator on the trapezius was set at $70 \%$ strength of maximum muscle stretch, and the vibration frequency was set at $100 \mathrm{~Hz}$. In this vibration condition, the degree of shortening of saccadic reaction time was similar to that in the moderate contraction of the neck extensors.

To investigate the mechanism of brain activation using muscle vibration, it appears necessary to use electrophysiological techniques such as evoked brain potentials (Fujiwara et al., 2005). The problematic issue in that examination was that the electroencephalograms (EEGs) were contaminated by electromagnetic waves generated by the vibrator's electromagnetic motor. This problem was solved by using a hydraulic vibrator that we developed. In EEG measurements using an auditory stimulus, in order to reduce the sound noise from the motor, vibration simulation must be applied at frequencies as low as possible. In this situation, investigation into the effectiveness of the low-frequency vibration activation of the nervous system is also necessary. Moreover, the skin over the muscle was stimulated because vibration stimulation is applied percutaneously. In addition, the vibrators will apply pressure to the muscles during the stimulation, which leads to stimulation of sensory organs other than muscle spindles. Such sensory information from cutaneous regions of the foot evokes specific postural responses and the illusion of postural changes (Kavounoudias et al., 1998; Roll et al., 2002). Therefore, it is important to determine the origin of the brain activation caused by the vibration stimulation. The purpose of the present study was to elucidate the effects of vibration stimulation to the neck extensor muscles on shortening of saccadic reaction time, using a new hydraulic vibrator under the following conditions: (1) various vibration frequencies during sub-maximum muscle stretch, (2) maximum muscle stretch, (3) muscle pressure, (4) touch to the skin.

\section{Methods}

\section{Subjects}

Subjects were 6 men and 8 women, ranging in age from 19 to 44 years (mean 26.5; SD 6.5). In a preliminary experiment, we measured the saccadic reaction time during neck flexion and selected subjects who exhibited shortening of the reaction time. It was expected that vibration to the neck extensors would have a significant effect on saccadic reaction time in these subjects. We previously reported that the difference in the shortening of the saccadic reaction time associated with the activation of neck extensors depended on sports experience; about $10 \%$ of all subjects, who had never belonged to any sports club, did not exhibit this shortening (Fujiwara et al., 2006). No subjects reported any history of neurological or orthopedic impairments, and all provided informed consent to the experimental protocol, which was approved by our institutional ethics committee.

\section{Apparatus and data recording}

The outline of the experimental setup is shown in Fig. 1. Each subject sat on a steel-frame chair with their back resting against a vertical wall and their trunk and shoulders secured with three acrylic belts, two around the chest and shoulders in a cruciform configuration and another around the hips. To prevent the scapula moving in the downward direction when pressure was applied to the trapezius, the axilla was elevated slightly and fixed by a cotton band hanging from the steelframe. Furthermore, length of the band was regulated to avoid compressing the axillary nerve. The subjects kept their knees flexed at an approximate $90^{\circ}$ angle and rested their feet on a low table. Neck flexion angle was defined as the rotational angle of the tragus around the acromion in the sagittal plane. The neck flexion angle was the same as that measured during quiet sitting posture, using an angular detector in which the center point was set at the acromion and the distance between the acromion and the tragus was regulated. The rotational angle of the head was also the same as that during the sitting posture, the purpose being to maintain constancy in sensory stimulus from the vestibular organ. To determine the head rotational angle, an angular detector using the principle of a pendulum was placed on the temple. A plate set against the temporal portion of the head was used to prevent horizontal head movement, and a vertical board placed against the occipital region was used to prevent extensional rotation of the head and neck. A chin stand was used to support the subject's head to help relax the neck extensor muscles as much as possible.

To monitor the electromyogram (EMG) of the trapezius muscles, the signal from the bipolar surface electrodes placed over the upper trapezius was amplified $(\times 2000)$ and band-pass filtered $(1.6 \mathrm{~Hz}-1.5 \mathrm{kHz})$ with an EMG amplifier (6R12, NEC, Japan). The electrode-input impedance was reduced to less than $2 \mathrm{k} \Omega$.

Since the two metal arms fixed to the frame each had two ball joints, the vibrator (EA-02, Electro Design, Japan) attached to the arm could be set freely in three dimensional space. The vibrator was driven by hydraulic pressure. To vibrate the upper trapezius muscles, the head of each vibrator was applied to the skin over a central region of the muscle (Fig. 1). The vibratory direction was set perpendicularly against the long axis of the muscle. Thereafter, we extended 


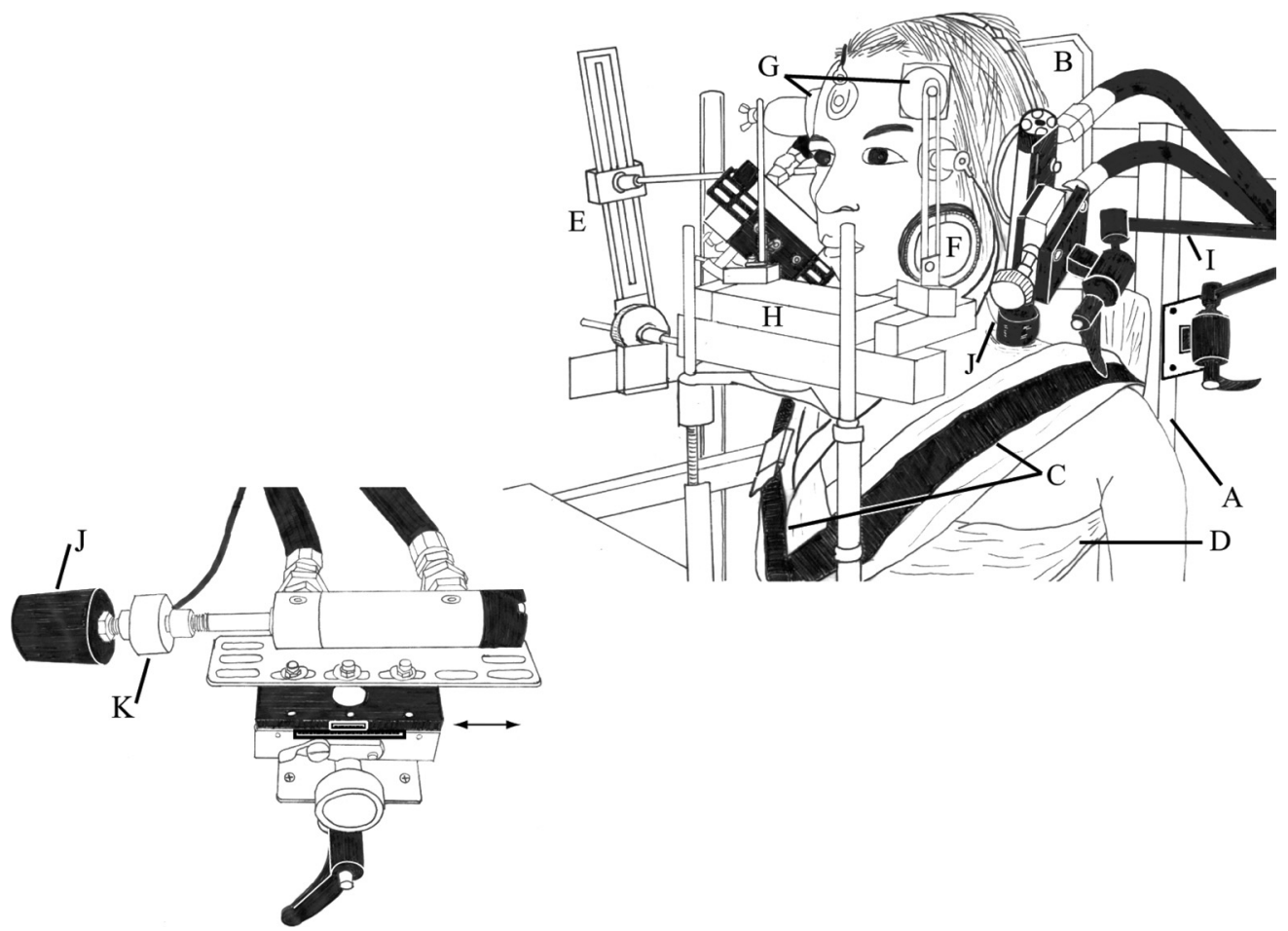

Fig. 1 Experiment setup. A vibrator with a strain-gauge transducer moves in the directions indicated by an arrow. A: Steel-frame, B: Vertical board, C: Acrylic belts, D: Cotton band, E: Angular detector for neck flexion, F: Angular detector for head rotation, G: Plates set at the temporal head, H: Chin stand, I: Metal arms, J: Hydraulic vibrator, K: Strain-gauge transducer

the trapezius muscles by regulating the length of a guide bar attached to the head of the vibrator (Fig. 1). Pressure on the trapezius was detected as the signal from the strain-gauge transducer (LU-5KSB34D, Kyowa, Japan) connected to the vibrator.

A visual stimulator (SLE-5100, Nihon Kohden, Japan) was used to induce saccadic eye movement. Visual stimuli were produced by alternately lighting the left and right light emitting diodes (LEDs) for random durations of 2-4 s using a function generator (WF1966, NF, Japan). Two LEDs were placed at the height of the nose root, and the distance between the central point of the two LEDs and the nose root was set at $50 \mathrm{~cm}$. The visual angle was set at $20^{\circ}$ and thus the LEDs were located $10^{\circ}$ left and right of the central point.

Horizontal eye movement was measured using the electrooculogram (EOG) technique. Surface electrodes were placed at the outer canthus of each eye with a ground electrode at the center of the forehead. Electrode-input impedance was reduced to less than $10 \mathrm{k} \Omega$. The signal from the electrodes was amplified $(\times 2000)$ using a DC amplifier $(A N-601 G$, Nihon Kohden, Japan). To obtain a steady EOG, the time interval from the point of securing the electrodes to the data recording was set at over $20 \mathrm{~min}$.

The signals from the visual stimulus and the electrodes were stored using a digital tape recorder (RD-130TE, Teac, Japan).

\section{Procedure}

Prior to the start of data recording, in order to help relax the trapezius muscles, contraction and relaxation of the shoulder girdle elevator muscles were alternately repeated several times and a deep breath was taken. The experimenter verbally instructed the subjects to relax the trapezius muscles, and this relaxation was monitored by EMG. Furthermore, a trial series in which subjects followed the alternately lit LED was carried out three times for 20 seconds each.

At first, the saccadic reaction time was measured under the resting condition, and thereafter it was measured under various vibration conditions.

The vibration conditions were as follows:

1) Sub-maximum muscle stretch condition

Prior to the measurement, the pressure on the trapezius was measured when the muscle was horizontally extended for as long as possible without vibration (right: $31.7 \pm 7.3 \mathrm{~N}$, left: $32.3 \pm 6.9 \mathrm{~N}$ ). Approximately $70 \%$ (right: $22.3 \pm 5.0 \mathrm{~N}$, left: $22.7 \pm 4.8 \mathrm{~N}$ ) of maximum pressure was applied to the trapezius muscles and then the vibration stimulation was applied to the muscles. To examine the optimal vibration frequency for the shortening of the saccadic reaction time, four vibration frequencies $(40,60,80$, and $100 \mathrm{~Hz})$ were used. Considering previous findings (Roll et al., 1990), the vibration amplitude was set at $0.5 \mathrm{~mm}$. For all vibration frequencies, we checked the noise during the vibration stimulation and made sure it was relatively low (no vibration: $43 \mathrm{~dB}, 40 \mathrm{~Hz}$ : $59 \mathrm{~dB}$, 
$60 \mathrm{~Hz}: 62 \mathrm{~dB}, 80 \mathrm{~Hz}: 67 \mathrm{~dB}$, and $100 \mathrm{~Hz}: 75 \mathrm{~dB}$ ).

Under the conditions described below, $60 \mathrm{~Hz}$ frequency was used.

2) Maximum muscle stretch condition

Vibration stimulation was applied to the trapezius muscles, which were extended for as long as possible in the horizontal direction without pain.

\section{3) Muscle pressure condition}

Pressure was applied to the trapezius muscles, directed vertically toward the scapula, and then the vibration stimulation was conducted. As in the sub-maximum muscle stretch condition, the pressure in this condition was approximately $70 \%$ of maximum pressure.

4) Touch condition

Vibration stimulation was applied to the same site as in the muscle stretch condition. In this condition, the pressure to the skin was set at $1 \mathrm{~N}$. This pressure was used as touch stimulation to the skin, referring to research by Martin and Park (1997) and Kennedy and Inglis (2002). The pressure applied to the finger flexors to evoke the tonic vibration reflex was $4 \mathrm{~N}$, and that applied to the sole to stimulate the cutaneous mechanoreceptors was less than $3 \mathrm{~N}$.

The reaction time was measured during a $30 \mathrm{~s}$ period and the measurement in each condition was carried out twice with a 3 min rest between each measurement. The measurement sequence among vibration conditions was randomly set for each subject. A 10 min rest was taken between the conditions. For all vibration conditions, measurement started 5 seconds after the stimulation began.

\section{Data analysis}

The data from EOG and the visual stimuli in the measurement of saccadic reaction time were sent to a computer (PAEX1524CWE, Toshiba, Japan) via an A-D converter at $1,000 \mathrm{~Hz}$ with 16 -bit resolution. Saccadic reaction time was defined as the latency to the beginning of eye movement following the onset of target movement (Fig. 2). In all conditions, measurement of saccadic reaction time was identified by visual inspection. When the start time of eye movement could not be measured because the EOG base line fluctuated, mean EOG amplitude was computed for a period of -300 to $-150 \mathrm{~ms}$ with respect to the onset of target movement and that value was adopted as the baseline. In this situation, start time was defined as when the EOG amplitude deviated more than the baseline $+2 \mathrm{SD}$ after $+100 \mathrm{~ms}$ with respect to the onset of target movement. Processing of reaction times was performed using BIMUTAS-II (Kissei Comtec, Japan).

\section{Statistical analysis}

One-way analysis of variance (ANOVA) with repeated measures was used to study the effect of the experimental condition on saccadic reaction time. Post-hoc analysis using Student-Newman-Keuls multiple comparison was performed to explore the nature of differences indicated by the analysis of variance. Alpha level was set at $p<0.05$. All statistics were

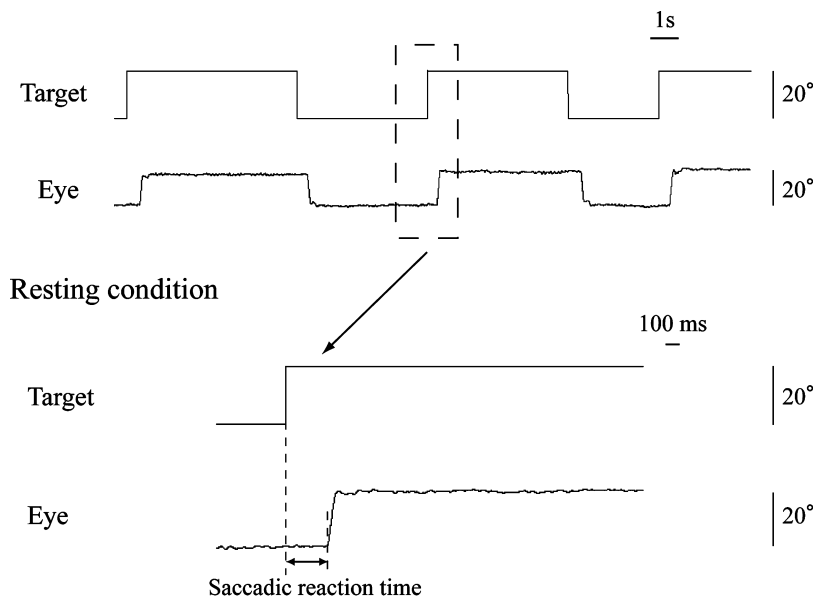

Sub-maximum muscle stretch condition $(60 \mathrm{~Hz})$

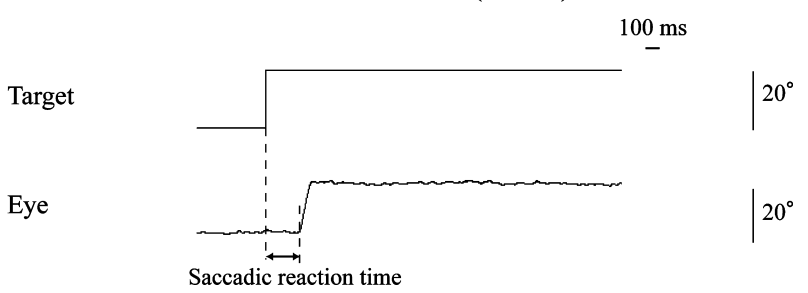

Fig. 2 Analysis of saccadic reaction time in sample records in the resting and sub-maximum muscle stretch condition (upper: successive recordings, middle: resting condition, lower: sub-maximum muscle stretch condition).

calculated using Excel 2000 (Microsoft, USA) with StatMate III (ATMS, Japan).

\section{Results}

The mean and standard deviation of saccadic reaction time in each condition are shown in Fig. 3. The saccadic reaction time under the resting condition was $197.5 \pm 18.9 \mathrm{~ms}$. The saccadic reaction times for $60 \mathrm{~Hz}, 80 \mathrm{~Hz}$, and $100 \mathrm{~Hz}$ vibration frequencies under the sub-maximum stretch condition were significantly shorter than that under the resting condition $\left(\mathrm{F}_{7,91}=9.09, \quad P<0.001 ; \quad 60 \mathrm{~Hz}: \quad 180.2 \pm 16.6 \mathrm{~ms} ; \quad 80 \mathrm{~Hz}\right.$ : $\left.182.2 \pm 15.5 \mathrm{~ms} ; 100 \mathrm{~Hz}: 179.5 \pm 15.3 \mathrm{~ms}, P_{s}<0.01\right)$. However, there was no significant difference among these three vibration conditions. The saccadic reaction time under the maximum muscle stretch condition $(195.3 \pm 20.1 \mathrm{~ms})$, the muscle pressure condition $(196.7 \pm 22.9 \mathrm{~ms})$, and the touch condition $(198.9 \pm 17.5 \mathrm{~ms})$ did not differ significantly compared with that under the resting condition.

\section{Discussion}

When vibration stimulation was applied to the trapezius muscles in the sub-maximum muscle stretch condition, the saccadic reaction time decreased for all vibration frequencies $(60,80$, and $100 \mathrm{~Hz})$ except $40 \mathrm{~Hz}$. Moreover, no significant difference in reaction time was found between the maximum 


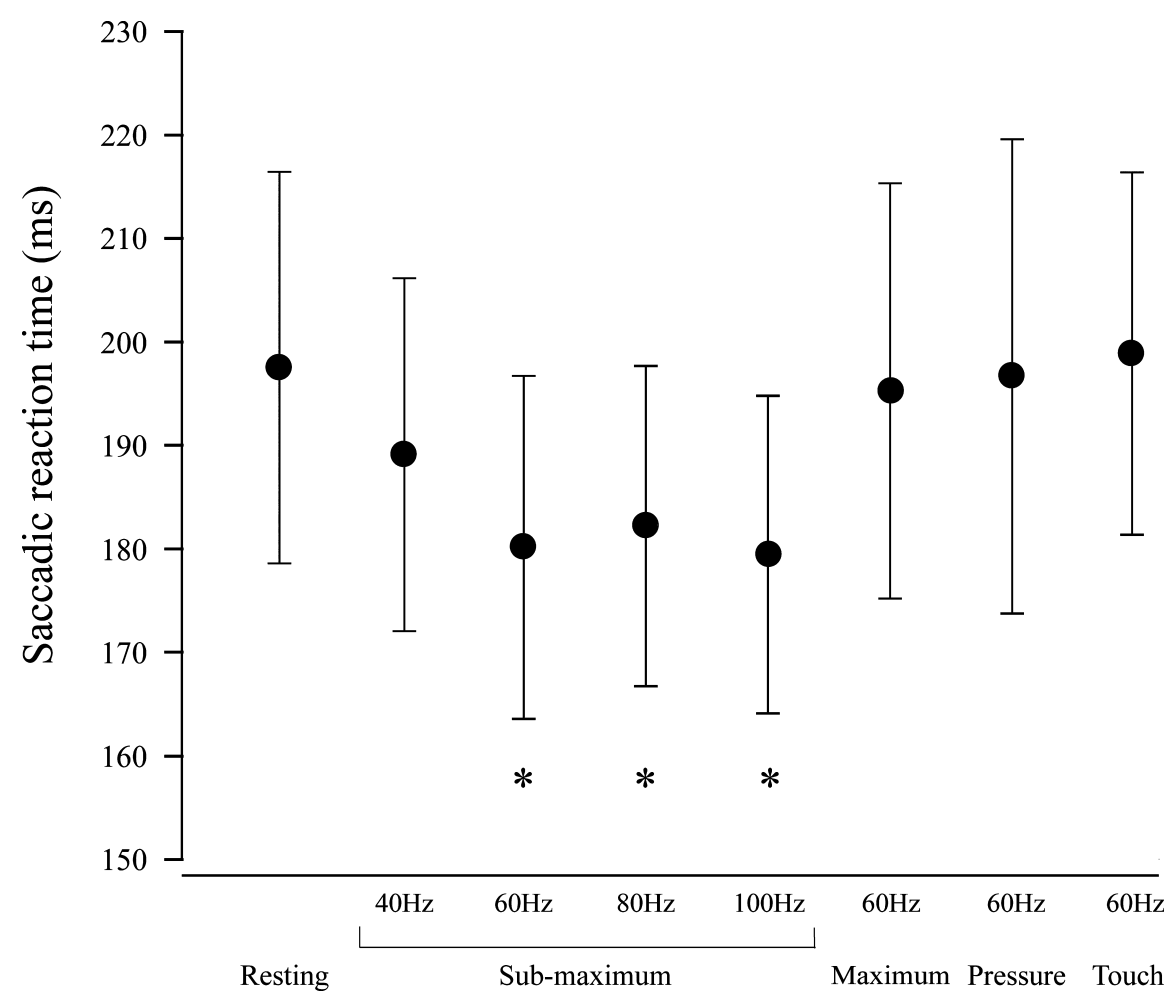

Fig. 3 Mean and standard deviation of saccadic reaction time in each condition. Asterisks indicate significant difference between the resting condition and another condition $(p<0.01)$.

muscle stretch condition and the resting condition. It has been reported that, when muscle length is less than maximum, sensory information from the muscle increases in response to vibratory stimulation (Eklund and Hagbarth, 1966; Burke et al., 1976a, b). Eklund and Hagbarth (1966) reported that the tonic vibration reflex during the vibration stimulation ceased when muscle length is maximum. They presumed that this inhibitory phenomenon was caused by sensory information from Golgi tendon organs. The firing ratio of the tendon organ associated with vibration stimulation increases in proportion to the increase in muscle tension (Giszter and Kargo, 2002). When vibration stimulation is applied to the stretched muscle, the firing ratio of the muscle spindle is saturated at resting length $+5 \%$ (Gister and Kargo, 2002). In the present study, the result during vibration stimulation in the maximum length condition indicated that the inhibitory effect on the central nervous system is caused by sensory information from the tendon organ and/or that the activation effect on the nervous system was not generated because the muscle spindle length did not change sufficiently.

No significant difference in the saccadic reaction time was observed among 60, 80, and $100 \mathrm{~Hz}$. It has been suggested that the firing ratio of the muscle spindle and excitability in the cerebral-spinal neural pathway increase according to the vibration stimulation frequency (Roll and Vedel, 1982; Steyvers et al., 2003). On the other hand, the vibration frequency that elicits the maximum firing ratio of the muscle spindle varies depending on the nerve ending, and at higher frequencies $(80-180 \mathrm{~Hz})$ the firing ratio rapidly decreases (Roll et al., 1990). Roll and Vedel (1982) found that at frequencies higher than $80 \mathrm{~Hz}$, the velocity of illusory movement during vibration stimulation is gradually reduced. This suggested that the processing capacity in the central nervous system was saturated due to high frequency activity of numerous muscle afferents activated synchronously. The frequency indicating the highest density of EMG power spectrum is consistent with the vibration stimulation frequency up to $100 \mathrm{~Hz}$; however, beyond this, the density of EMG in the vibration frequency gradually reduces according to the vibration frequency, and subharmonic synchronized EMG becomes predominant (Martin and Park, 1997). As a result of the combination of stretch and vibration effects, the maximum effect on the shortening of the saccadic reaction time might therefore reach a plateau at $60 \mathrm{~Hz}$ and be maintained until $100 \mathrm{~Hz}$.

The noise of the apparatus was lower when the vibration frequency was lower; therefore, the finding that a sufficient activation effect was obtained at $60 \mathrm{~Hz}$ vibration has important implications, particularly in examinations related to auditory sense.

It has been reported that sensitivity to vibration stimulation is very high in the baroreceptors (Roll and Vedel, 1982). Therefore, it is assumed that the baroreceptors in the neck extensors participate in the shortening of the saccadic reaction time during vibration stimulation to the neck extensors. However, no significant difference in the reaction time was found between the muscle pressure condition and the resting 
condition. This finding suggests that saccadic reaction time is not influenced by sensory information from the baroreceptors. Furthermore, no significant difference in the reaction time was found between the touch condition and the resting condition. Another study also reported that an increase in sensory information from the muscle is not observed in vibratory stimulation to the skin (Roll and Vedel, 1982). The results of the present study strongly suggest that the important factor for shortening of the saccadic reaction time was the increase in muscle afferent information during vibration stimulation to the neck extensors.

The important findings in the present study were that vibration stimulation to the trapezius at frequencies of $60-100 \mathrm{~Hz}$ at $0.5 \mathrm{~mm}$ amplitude in the sub-maximum stretch condition effectively shortened saccadic reaction time. The main underlying mechanism appears to be Ia information originating from muscle spindles.

\section{References}

Berthoz A (1996) The role of inhibition in the hierarchical gating of executed and imagined movements. Brain Res Cogn Brain Res 3: 101-113

Burke D, Hagbarth K-E, Löfstedt L, Wallin BG (1976a) The responses of human muscle spindle endings to vibration of non-contracting muscles. J Physiol (Lond) 261: 673-693

Burke D, Hagbarth K-E, Löfstedt L, Wallin BG (1976b) The responses of human muscle spindle endings to vibration during isometric contraction. J Physiol (Lond) 261: 695-711

Eklund G, Hagbarth K-E (1966) Normal variability of tonic vibration reflexes in man. Exp Neurol 16: 80-92

Fujiwara K (1994) 'The ready' posture and the speed of responding movements. Jpn J Sports Sci 13: 739-749 [In Japanese]

Fujiwara K, Kunita K, Toyama H (2000) Changes in saccadic reaction time while maintaining neck flexion in men and women. Eur J Appl Physiol 81: 317-324

Fujiwara K, Kunita K, Toyama H, Miyaguchi A (2001) Saccadic reaction times during isometric voluntary contraction of the shoulder girdle elevators and vibration stimulation to the trapezius. Eur J Appl Physiol 85: 527-532

Fujiwara K, Shigeiwa M, Maeda K, Kunita K (2005) Changes in posterior auricular muscle response and middle-latency brain potential evoked by auditory stimuli according to neck flexion. Health Behav Sci 3: 69-77

Fujiwara K, Kunita K, Watanabe H (2006) Sports exercise effect on shortening of saccadic reaction time associated with neck extensor muscle activity. Int J Sports Med (in press)

Fukuda T (1957) Stato-kinetic reflexes in equilibrium and movement. Igaku-Shoin, Tokyo [In Japanese]

Giszter SF, Kargo WJ (2002) Separation and estimation of muscle spindle and tension receptor populations by vibration of the biceps muscle in the frog. Arch Ital Biol 140: 283294
Grantyn A, Berthoz A (1987) Reticulo-spinal neurons participating in the control of synergic eye and head movements during orienting in the cat. I. behavioral properties. Exp Brain Res 66: 339-354

Hagbarth K-E, Vallbo ÅB (1968) Discharge characteristics of human muscle afferents during muscle stretch and contraction. Exp Neurol 22: 674-694

Hinoki M, Hine S, Okada S, Ishida Y, Koike S, Shizuku S (1975) Optic organ and cervical proprioceptors in maintenance of body equilibrium. Acta Otolaryngol Suppl 330: $169-184$

Howorth B (1946) Dynamic posture. J Am Med Assoc (Chicago) 131: 1398-1404

Kavounoudias A, Roll R, Roll JP (1998) The plantar sole is a 'dynamometric map' for human balance control. Neuroreport 9: 3247-3252

Kennedy PM, Inglis JT (2002) Distribution and behaviour of glabrous cutaneous receptors in the human foot sole. J Physiol (Lond) 538: 995-1002

Kinugasa T, Fujita T, Tanaka H (1985) Effect of preliminary jump action on choice response time. Jpn J Phys Educ 24: 301-311 [In Japanese]

Kunita K, Fujiwara K (1996) Relationship between reaction time of eye movement and activity of the neck extensors. Eur J Appl Physiol Occup Physiol 74: 553-557

Martin BJ, Park HS (1997) Analysis of the tonic vibration reflex: influence of vibration variables on motor unit synchronization and fatigue. Eur J Appl Physiol Occup Physiol 75: 504-511

Roll JP, Vedel JP (1982) Kinaesthetic role of muscle afferents in man, studied by tendon vibration and microneurography. Exp Brain Res 47: 177-190

Roll JP, Gilhodes JC, Roll R, Velay JL (1990) Contribution of skeletal and extraocular proprioception to kinaesthetic representation. In Jeannerod $\mathrm{M}$ ed. Attention and performance XIII: motor representation and control. Erlbaum Associates, New Jersey, 549-566

Roll R, Kavounoudias A, Roll JP (2002) Cutaneous afferents from human plantar sole contribute to body posture awareness. Neuroreport 13: 1957-1961

Shimura K, Kasai T (2002) Effects of proprioceptive neuromuscular facilitation on the initiation of voluntary movement and motor evoked potentials in upper limb muscles. Hum Mov Sci 21: 101-113

Steyvers M, Levin O, Verschueren SM, Swinnen SP (2003) Frequency-dependent effects of muscle tendon vibration on corticospinal excitability: a TMS study. Exp Brain Res 151: 9-14

Taniguchi R, Nakamura R, Yokochi F, Narabayashi H (1980) Effects of postural change of the shoulder on EMG reaction time of triceps brachii. Appl Neurophysiol 43: 40-47

Vallar G, Rusconi ML, Barozzi S, Bernardini B, Ovadia D, Papagno C, Cesarani A (1995) Improvement of left visuospatial hemineglect by left-sided transcutaneous electrical stimulation. Neuropsychologia 33: 73-82 
Received: May 15, 2006

Accepted: August 3, 2006

Correspondence to: Katsuo Fujiwara, Department of Human Movement and Health, Graduate School of Medical Science,
Kanazawa University, 13-1 Takara-machi, Kanazawa 9208640, Japan

Phone: +81-76-265-2225

Fax: +81-76-234-4219

e-mail: fujikatu@med.kanazawa-u.ac.jp 